\title{
Interactive comment on "Changes in the shape of cloud ice water content vertical structure due to aerosol variations" by Steven T. Massie et al.
}

\section{Anonymous Referee \#2}

Received and published: 14 February 2016

This study addresses the question of effect of aerosols on the invigoration of deep tropical clouds. They regard an enhancement of the ice water content (IWC) as an indicator for the invigoration. While invigoration can certainly increase the IWC, the IWC was shown to be potentially enhanced also by microphysical effects of the added aerosols. Fan et al. (2013) showed that larger numbers of smaller ice particles fall more slowly and therefore enhance the IWC. This has to be added to introduction.

Major comments:

1. The main response parameters are poorly defined. Please define clearly IWCsum, IWCreg and IWCshape with equations and allocate for that explanation a figure with illustration. Please state the units.

2. The authors conclude that cloud adjacency does not affect much their conclusions. 
The data that they show to support that is not very convincing. The obvious way to show this is to repeat the final results for the various adjacency thresholds, and see the extent that it affects these results. Sample size should not be an issue for the final results shown in Figures 8 and 9.

3. It is not clear to me how Figure 1 was constructed. It appears that more profiles were averaged towards the larger numbers on the abscissa, because they converge towards ordinate value of zero. How was that done? Were the profiles binned and averaged in some way?

4. The authors state that "three AOD bins (i.e. $0.05-0.15,0.15-0.35,0.35-0.45$ ), were chosen to represent low, medium, and high amounts of AODs". By doing this the authors ignored the two main properties of aerosol effects on cloud invigoration, as described in two papers in Science: (Koren et al., 2008; Rosenfeld et al., 2008). First, the cloud invigoration responds to the logarithm of the aerosols concentrations. Second, the effect saturates at AOD of 0.25 to 0.3 , and may reverse at larger AOD. This is evident in Figure 3, where there is large difference between the lines of AOD 1 and 2, but bins 2 and 3 are practically the same. The authors have to expand the introduction to include the discussion of the aerosols effect, as mentioned here. Furthermore, the analysis has to be redone with re-binning accordingly. The cases with $A O D<0.05$ are the cleanest and thus expected to have the greatest contrast to the polluted cases. Based on the principle of the logarithmic effect, the difference between $A O D<0.05$ and AOD of 0.05 to 0.15 should be larger than what the authors found between bins 1 and 2 in the present version,. Why did the authors exclude $A O D<0.05$ ? This should be a bin on its own, which I expect to be the most informative. In summary, for the paper to be considered for publication in ACP it has to undergo a major revision. The background has to be rewritten with a more physical basis, the methodology has to be clarified, the analyses have to be completely redone with new binning, the effects of adjacency effect have to be tested on the final results, and the discussion of the results has to commensurate with the newly written physical background. 
I did not comment on minor issues, because they likely will not survive the revision, if done as I expect it to be.

\section{References:}

Fan J., L. R. Leung, D. Rosenfeld, Q. Chen, Z. Li, J. Zhang, H. Yan, 2013: Microphysical effects determine macrophysical response for aerosol impacts on deep convective clouds. Proceedings of the National Academy of Sciences, 110(48), E4581-E4590.

Koren, I., Martins, J. V., Remer, L. A., \& Afargan, H. (2008). Smoke invigoration versus inhibition of clouds over the Amazon. science, 321(5891), 946-949.

Rosenfeld, D., Lohmann, U., Raga, G. B., O’Dowd, C. D., Kulmala, M., Fuzzi, S., ... \& Andreae, M. O. (2008). Flood or drought: how do aerosols affect precipitation?. science, 321(5894), 1309-1313.

Interactive comment on Atmos. Chem. Phys. Discuss., doi:10.5194/acp-2015-732, 2016. 